\title{
Results of internal fixation and anterior muscle pedicle bone grafting in old femoral neck fracture MI Khalil', A Rahman ${ }^{2}$
}

\begin{abstract}
Sixteen cases of old fracture neck femur were treated by internal fixation and anterior muscle pedicle bone grafting of rectus femoris, sartorius bone block from anterior superior iliac spine. The treatment period were from January 2000 to December 2009 at Khulna Medical College Hospital and some private hospital. Different methods of fixation and bone grafting procedure were reported. We did this procedure by open reduction and internal fixation by cannulated hip screws and muscle pedicle bone grafting. Additional cancellous bone grafting were done in every cases. Follow up period was 2 to 7 years (average 5 years). Evaluation parameter were union, non union. collapse of neck, avascular necrosis of femoral head (AVN) coxavara, shortening, pain, range of movement and functional activities and over all satisfaction of patient. The results of fracture healing rate was good (9), fair (4) and poor (3). The technique is simple, rewarding and easy access of fixation and muscle pedicle bone grafting. Bone graft was placed by making a gutter at fracture site and maintained by a screw or prolin suture.
\end{abstract}

Bang Med J (Khulna) 2010; $43: 10-11$

\section{Introduction}

Femoral neck fracture is a common fracture. The treatment of femoral neck fracture is still a matter of controversy even after so many recent advancement in orthopaedics. Improper treatment causes non union, avascular nurosis (AVN) and severe joint dysfunction. In particular femoral neck fracture in young adults, according to karliff and protzwan reported that the incidence of non union can be as high as 59\%. In order to reduce the incidence of non union, Judet first reported in 1992 that after fixation with muscle pedicle bone graft, the treatment of fracture neck femur has improved significantly. The treatment of femoral neck fracture is still unsolved problem. Non union and AVN are two main complications of this fracture 'especially if patients present late. Muscle pedicle bone grafting has been advocated to provide additional blood supply. The rate of non union has been reduced by anatomical reduction and stable fixation of fracture but the incidence of AVN is evident. In 1962, the autogenous muscle pedicle bone graft based on the Quadratus femoris muscle was used for the lst time. Later fresh autogenous cancellous iliac bone chips combined with muscle pedicle bone grafting have been reported to be good.

\section{Materials \& Methods}

Open reduction and internal fixation with muscle pedicle bone grafting was done in 16 cases from Jan2000 to Dec. 2009 at KMCH and some private hospital. Age of the patient from 20 years to 50 yrs. (average 35yrs). Duration of fracture period from 2 months to 6 months (average 3 months) Male female ration 10:5. Evaluation parameter were union, Non union, collpase of Neck, Coxavara, AVN of femoral head, pain, shortening. Range of movement and functional activity.

In all the patient preoperative anterioposterior radiograph of the hip was taken. All the patients were operated upon a supine position on a fracture table. An anteriolateral approach was used to expose the fracture site. Reduction of the fracture was by leg traction and manipulation. A check X-ray of anterioposterior and lateral radiograph was taken to confirm reduction and the position of the implants. Fixation was done by compression hip screws with anterior muscle pedicle bone grafting. Supplementary autograft from anteriorsuperior iliac crest were taken.

\section{Results}

The results were assessed in terms of osseous union. There was clinical as well as radiological union in 13 cases which could be followed. But in 3 cases were non union and AVN of femoral head. osseous union and AVN were two major parameter to assess the results. There were 11 male and 5 female patients. Follow up range from 2-7 years (average 5 yrs). There were 9 cases with good functional results and had the ability to squat and site crosslegged (Table I), fair in 4 cases and poor results in 3 cases. Complication were AVN. Coxavara. Collapsed femoral neck and Non union. Muscle pedicle bone grafting with internal fixation is a viable treatment option in femoral neck fracture with late presentation.

1. Md Ibrahim Khalil M.S (Orth), Senior Consultant, Dept of Orthopaedics, Khulna Medical College Hospital

2. Ashiqur Rahman MBBS, Asstt, Registrar, Dept of Orthopaedics, Khulna Medical College Hospital 
Table I

Results of 16 case

\begin{tabular}{cll}
\hline Number & Result & Feature \\
\hline good (9) & Union & $\begin{array}{l}\text { Full range of movement \& functional activity } \\
\text { to squat \& cross legged. No pain, no AVN } \\
\text { and no coxavara, No shortening. }\end{array}$ \\
fair (4) & $\begin{array}{l}\text { Union } \\
\text { satisfactory }\end{array}$ & $\begin{array}{l}\text { Mild limitation of movement and squating. } \\
\text { mild pain \& mild coxavura } \\
\text { No AVN. } \\
\text { Noon union, painful movement, collapse of } \\
\text { femoral neck. } \\
\text { AVN of femoral Head }\end{array}$ \\
\hline
\end{tabular}

\section{Discussion}

The treatment of femoral neck fracture is still a matter of controversy even after so many recent advancements in orthpaedics. Non union and AVN are the two main complication of this fracture. The rate of non union has been reduced by anatomical reduction and stable fixation of fracture but the incidence of AVN is evident. In 1962, the autogenous muscle pedicle graft based on the quadratus femosis muscle was done for the first time.2 Late, fresh autogenous cancellous iliac bone chips combined with muscle pedicle bone grafting have been reported to be good.3 There have been encouraging reports by using similar technique. 4

In femoral neck fracture, bone grafting is the scope of fracture healing process, only dependent on the role of bone marrow into the bone to complete. So the blood supply of fracture and its stability during the healing process will be decisive factor. According to MeiFang Fuil such as healing of femoral neck fracture and femoral head vitality ultrastructure study confirmed that the femoral head after femoral neck fracture severely damage the blood supply.

Anatomical reduction, impaction and rigid internal fixation are essential in treating femoral neck fracture. Muscle pedicle bone grafting has been advocated by many investigators along with rigid internal fixation to prevent Nonunion and AVN of femoral head. This provides an additional source of blood supply for the femoral head which may have been rendered ischaemic by the fracture and also allows reduction and impaction of the fracture under direct vision. We have also used cancellous bone chips to fill up any gap at the fracture site before internal fixation is done. This increases the stability of the fracture reduction. Reduction of blood supply of fracture of femoral head depends upon the type of fracture and age of fracture. Stable and reliable fixation are closely related to AVN and blood supply of femoral head.

The postoperative mode of immobilization depended upon the rigidity of the fixation and the age of the patient. Single hip spica was used in all the cases and average period of immobilization in the postoperative period was 8 wks. Partial weight bearing was allowed gradually depending upon the status of union. Full weight bearing was allowed only after full osseous union on an average of 1 year after the operation.

\section{Conclusion}

We conclude that muscle pedicle bone grafting with interval fixation is a very rewarding method of treatment of femoral neck fracture with late presentation. This treatment can promote the fracture healing and reduce the incidence of avascular necrosis. This kind of surgery can improve the union of fracture and reduce osteonecrosis incidence of the femoral head.

\section{References}

1. Garden RS. Reduction and fixation of the subcapital fractures of the femur. Orthop Clin North Am 1974; 5: 683-712

2. Judet R. Treatment des fractures ducol du femur par graffe pediculee. Acta Orthop Scand. 1962; 32: 421-7.

3. Meyers MH, Harvey JP, Jr, Moore TM. Treatment of displaced subcapital and transcervical fractures of the femoral neck by muscle neck by muscle pericle bone graft and internal fixation, a preliminary report on one hundred and fifty cases. J Born Joint Surg Am 1973; 55: 257-74.

4. Baksi DP. Internal fixation of un-united femoral neck fractures combined with muscle pedicle bone grafting. J Bone Joint Surg Pr. 1986; 68: 239-45.

5. Salvati EA. Wilson PD., Jr Long term results of femoral head replacement. J Bone Joint Surg Am 1973; 55: 516-24.

6. Koval, Zuckeman Hip fractures: A practical guide to management. New York: Springer Verlag Inc; 2005.

7. Schatzker, Tile Springar The rationale of operative fracture care, Vertage Berlin Heidelberg 3rd ed. 2005 p. 351.

8. Meirui Fang, WU Yuan-ching. Femoral neck fractuere and femoral head vitality of the ultrastructural changes. Chinese Journal of Trauma, 1996, 12, 240.

9. $\mathrm{Lu} \mathrm{Yu}$ Park. Practical Orthopedics. Beijing: People's Medical Publishing House, 1991; 644.

10. Wang Yongxi, Chen Rui, Zhang Da, et al. With femoral muscle pedicle graft and internal fixation of femoral neck fracture. Zhonghua Surgery, 1982, 20: 289

11. AK Gupta, Sanjai Rastogi, R Nath, Department of Orthopaedics, GSVM Medical College, Kanpur University, Kanpur, India. 2008; 42: 39-42. 